\title{
Emprego de bomba multifásica como unidade geradora de microbolhas de ar em sistema de flotação aplicado ao pré-tratamento de efluente têxtil
}

\author{
Multiphase pump as microbubble generator for flotation system \\ applied to pre-treatment of textile wastewater
}

\begin{abstract}
Andre Pioltine
Engenheiro Ambiental. Aluno regular de doutorado do Programa de Pós-Graduação em Engenharia Hidráulica e Saneamento pela Escola de Engenharia de

São Carlos da Universidade de São Paulo (EESC/USP)
\end{abstract}

Marco Antonio Penalva Reali

Engenheiro Civil. Professor Doutor do Departamento de Hidráulica e Saneamento da EESC/USP

\section{Resumo}

O artigo apresenta os resultados de uma pesquisa desenvolvida com o objetivo de avaliar o desempenho de bomba multifásica (mistura de ar e água) aplicada em substituição à câmara de saturação, em sistema de flotação localizado em uma indústria têxtil. Na unidade piloto (taxa de aplicação superficial de clarificação igual a 128,6 \pm 10 m.d ${ }^{-1}$ ), foi verificado que os melhores resultados em termos de eficiência de remoção de turbidez e cor aparente (eficiência de remoção superior a 95\%) foram obtidos nos ensaios realizados com razão de recirculação em torno de 30\% associada à composição volumétrica de ar de 13\%.

Palavras-chave: flotação; bomba multifásica; tratamento de efluente têxtil.

\begin{abstract}
This paper presents the results of a research carried out that aims to evaluate the performance of a multiphase pump (mixture of air and water) applied to replace the saturator chamber in a flotation system located in a textile industry. In the pilot unit (hydraulic surface loading equal to $128.6 \pm 10 \mathrm{~m} . \mathrm{d}^{-1}$ ), it was verified that the best results in terms of removal efficiency of turbidity and apparent color (efficiency removal over 95\%) were obtained for the essays performed with recirculation ratio equal to $30 \%$ and air supply of $13 \%$.
\end{abstract}

Keywords: flotation; multiphase pump; treatment of textile effluent.

\section{Introdução}

Segundo Reali (1991), a flotação é um processo de separação de partículas sólidas ou líquidas de uma fase líquida com a utilização de bolhas de gás (normalmente o ar), as quais ao aderirem à superfície das partículas aumentam seu empuxo e provocam sua ascensão em direção à superfície da fase líquida, na qual serão devidamente coletadas por meio de dispositivos específicos.

A flotação por ar dissolvido (FAD) foi inicialmente utilizada na exploração de matérias-primas minerais no final do século 19, sendo introduzida como alternativa para o tratamento de águas residuárias na década de 1920 (KIURU, 2001). Desde então, importantes desenvolvimentos no processo foram verificados, os quais contribuíram significativamente para o aumento em sua utilização.
No tratamento de efluentes industriais, a FAD é o método mais utilizado, podendo ser incorporado aos processos de mineração e industrial das seguintes formas: (a) como um processo unitário (auxiliar ou principal) na remoção de contaminantes que não são separados por outras tecnologias; (b) na remoção de compostos orgânicos e resíduos químicos da água; (c) no processo de espessamento de lodo; entre outros (RUBIO; SOUZA; SMITH, 2002).

Entre os numerosos avanços nos últimos 20 anos, os quais têm contribuído para uma maior eficiência e uso da FAD nas aplicações industriais, pode-se citar os avanços nos métodos para a saturação de ar na água. Assim, dentre os novos métodos objetos de pesquisas recentes (por exemplo os estudos desenvolvidos por Han et al, 2007; Lee et al, 2007; Sohn et al, 2007), tem-se o emprego de bombas multifásicas em substituição aos sistemas de saturação tradicionais. Estes 
são formados por câmara de saturação, compressor de ar e bomba centrífuga de alta pressão.

De acordo com Ross, Smith e Valentine (2000), as bombas multifásicas apresentam diversas vantagens em relação aos sistemas de geração de microbolhas de ar tradicionais, os quais são constituídos de bombas centrífugas associadas aos compressores de ar e à câmara de saturação. Dentre tais vantagens, vale destacar: (1) alta eficiência volumétrica, fornecendo uma grande massa de ar por unidade de volume de recirculação; (2) eliminação da necessidade do uso de câmaras de saturação; (3) o suprimento de ar pode ser por meio de compressores de baixa pressão ou a partir do próprio ar atmosférico. Neste último caso, não há necessidade do uso de compressores de ar.

Com relação às suas desvantagens, Ross, Smith e Valentine (2000) citam: (1) maior requerimento de potência devido às altas pressões de operação; (2) o possível desgaste causado por partículas sólidas pode reduzir a efetividade da bomba com o tempo; (3) pode resultar em bolhas de tamanhos maiores, causadas pelo possível excesso de ar fornecido

Desta forma, em vista das potenciais vantagens em termos de simplicidade oferecidas pelas bombas multifásicas em comparação com as tradicionais câmaras de saturação, o presente trabalho apresenta como proposta central a avaliação do desempenho da bomba multifásica na geração de microbolhas de ar, em um sistema de flotação em escala piloto aplicado na pré-clarificação de efluente proveniente de indústria têxtil.

\section{Materiais e métodos}

O projeto foi dividido nas seguintes etapas: Etapa I - com o emprego do Flotateste, foram determinadas as dosagens adequadas de coagulante (LAB TAE 333 - Teor de $\mathrm{Al}_{2} \mathrm{O}_{3}$ igual a 16,0 - 20,0\%) e de auxiliar de floculação (LAB TAE 409 - Polímero Catiônico Pó) para o tratamento do despejo líquido industrial em estudo; Etapa II - utilizando-se uma unidade piloto de FAD montada nas instalações de uma indústria têxtil, avaliou-se a influência das condições de operação (taxa de aplicação superficial, razão de recirculação, pressão de saturação e quantidade de ar fornecida), na eficiência do sistema constituído pela bomba multifásica.

\section{Flotateste}

Equipamento em escala de laboratório e com alimentação por batelada, com quatro colunas de flotação independentes, construídas em acrílico e cada coluna possui um agitador removível, conforme proposto por Reali (1991). Estas funcionam como unidades de floculação e flotação. Interligada às quatro colunas de flotação há a câmara de saturação pressurizada, na qual o ar foi dissolvido durante os ensaios à pressão de 550k Pa (Figura 1).

\section{Unidade piloto de FAD}

A unidade piloto utilizada nesta pesquisa é composta por uma unidade de floculação mecanizada com dois compartimentos em série, associada a uma unidade de flotação, cujas dimensões são apresentadas na Figura 2. Cada compartimento de floculação possui um agitador, com quatro braços e duas paletas por braço. Na entrada da unidade de flotação, estão instalados quatro dispositivos (válvulas agulha) para a despressurização do efluente recirculado. A jusante deste ponto há a zona de contato, na qual ocorre o encontro entre as microbolhas de ar geradas após a despressurização da vazão de recirculação e os flocos formados nas câmaras de floculação. Logo após, encontra-se a zona de separação, que é a região onde ocorre a flotação propriamente dita. Nesta, a camada de lodo ascende à superfície sendo removida constantemente por raspadores específicos. Durante a operação do sistema, uma parcela do efluente pré-clarificado pela unidade piloto era recirculado através da bomba multifásica (sistema em estudo em substituição às câmaras de saturação convencionais), na qual era realizada a saturação do efluente com ar proveniente diretamente da atmosfera. Já a outra parcela era retornada ao tratamento de efluentes existente na indústria.

\section{Bomba multifásica}

Esta tecnologia surgiu com o objetivo de substituir o sistema convencional de geração de microbolhas de ar no sistema de FAD, o qual é usualmente constituído por câmara de saturação, compressor de ar e bomba centrífuga de alta pressão. As principais características da bomba multifásica em estudo são: a marca eraEDUR; o modelo, EB3u; a faixa de vazão era $0,5-7,0 \mathrm{~m}^{3} \cdot \mathrm{h}^{-1}$; o conteúdo em volume de gás era de até 15\% em relação à vazão de recirculação; a velocidade de rotação, $3.500 \mathrm{rpm}$; a potência nominal, 2,2 kW; a frequência era de $60 \mathrm{~Hz}$. As curvas características da bomba, fornecidas pelo fabricante, são apresentadas na Figura 3.

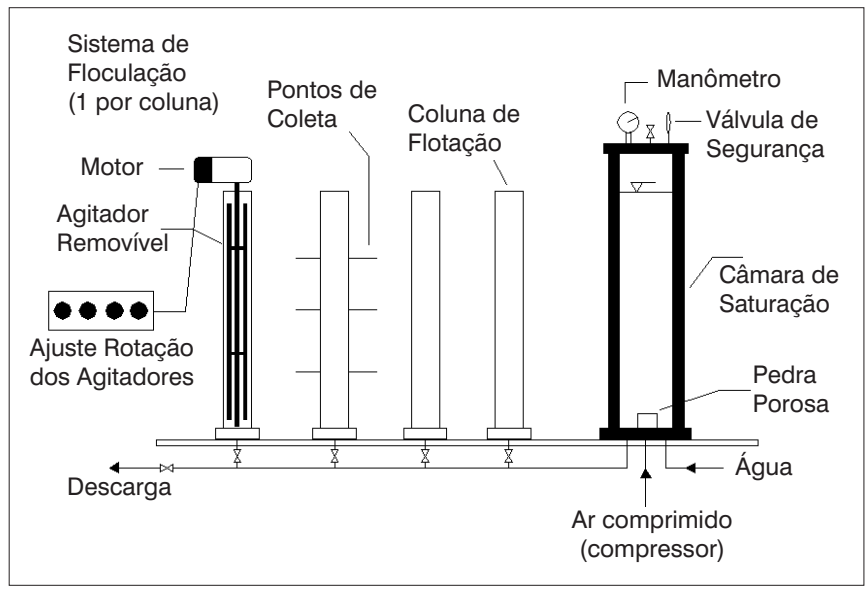

Figura 1 - Esquema ilustrativo do Flotateste utilizado para os ensaios em escala de laboratório (proposto por Reali, 1991) 


\section{Layout da instalação}

A Figura 4 apresenta o layout da instalação e os principais componentes utilizados nesta pesquisa. Pode-se verificar que o despejo líquido industrial em estudo foi bombeado do tanque de equalização (já existente na indústria) até a unidade piloto de flotação. No tanque de equalização, ocorreu a homogeneização e a correção de pH de todo despejo líquido produzido pela indústria. O processo de mistura rápida (aplicação de coagulante) ocorreu por meio de um injetor acoplado à tubulação (coagulação in-line). Após o ponto de aplicação do coagulante, instalou-se um medidor de vazão eletromagnético para o controle da vazão afluente ao sistema.

A aplicação do polímero ocorreu pelo manifold fixado na parte superior de uma abertura existente entre as duas câmaras de floculação, a qual permitiu a passagem do despejo líquido de uma câmara para a outra. Em seguida, o despejo líquido previamente coagulado e floculado era clarificado na unidade de flotação. Para o controle da parcela de efluente tratado recirculado e da quantidade de ar fornecida ao sistema, foram instalados um medidor de vazão eletromagnético e um microssensor, respectivamente. O medidor de vazão eletromagnético (linha de recirculação), por tratar-se de equipamento sensível, teve que ser posicionado na linha de sucção da bomba multifásica à montante do ponto de entrada de ar. Isto foi devido ao fato de que a bomba, em alguns ensaios realizados durante a pesquisa, não dissolveu completamente o ar no despejo líquido, proporcionando o surgimento de macrobolhas. Estas, ao passarem pelo medidor de vazão, provocavam leituras instáveis e errôneas.

Assim, com a finalidade de evitar que eventuais macrobolhas geradas pela bomba multifásica chegassem ao tanque de flotação, um dispositivo de expulsão de macrobolhas (DEM) foi inserido na linha de recirculação. Este dispositivo foi concebido de forma a se obter velocidade descendente da água em seu interior em torno de 18,7 \pm $6,2 \mathrm{~m} / \mathrm{h}$. Além disso, instalou-se uma ventosa na parte superior deste dispositivo, com o objetivo de expulsar o excesso de ar proveniente das possíveis macrobolhas geradas. Para a determinação da quantidade de ar liberada pelo DEM, utilizou-se outro dispositivo, conforme será comentado.
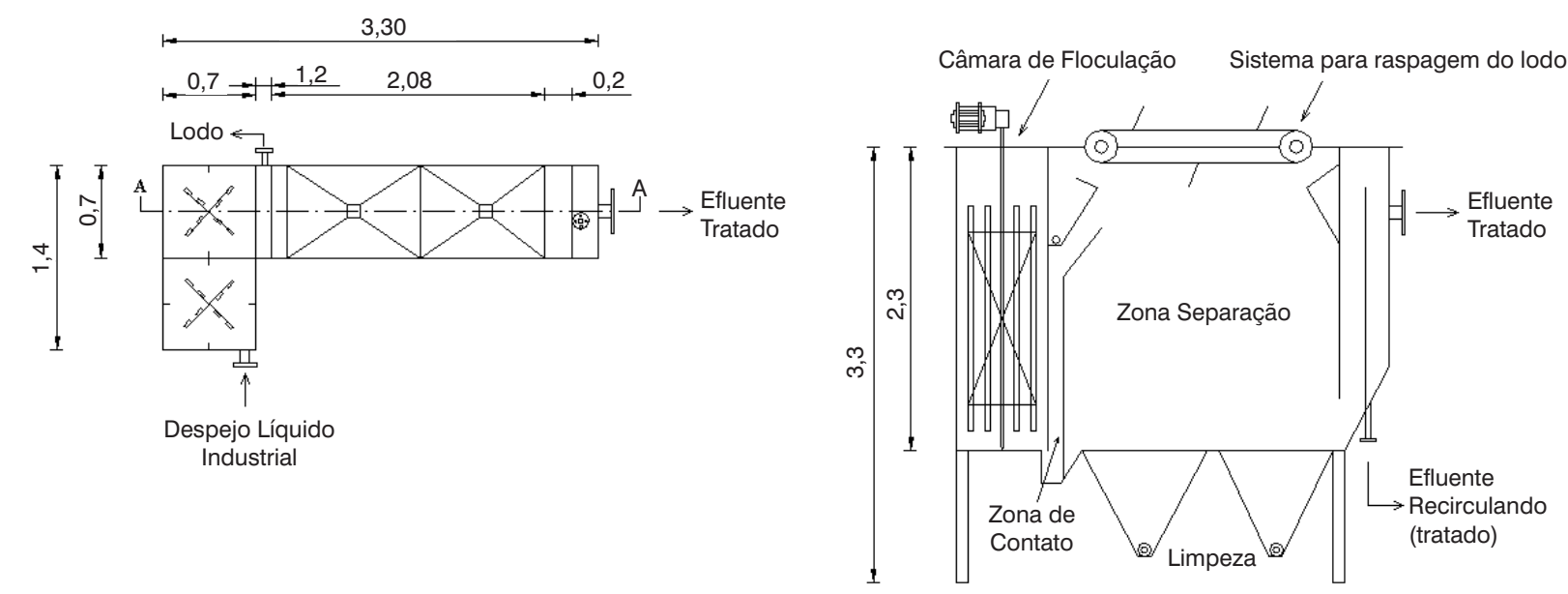

Figura 2 - Planta e corte A-A da instalação piloto de FAD (unidades em metro)

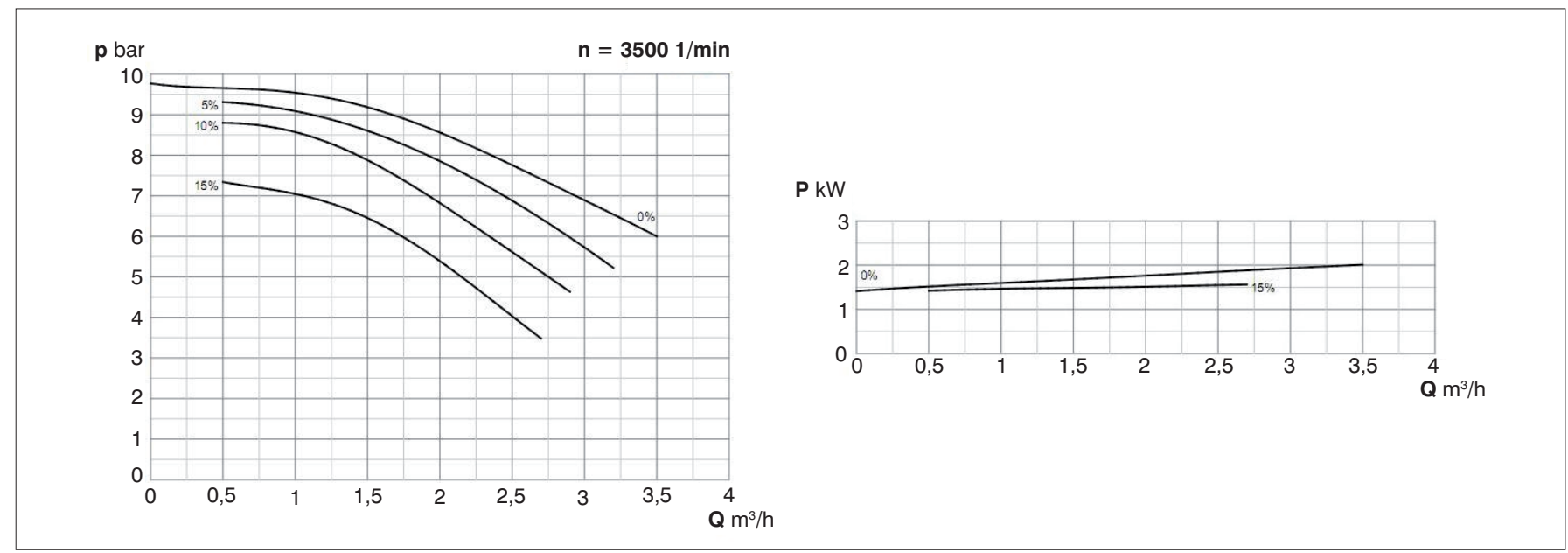

Figura 3 - Curvas características da bomba multifásica fornecidas pelo fabricante (Água: $\rho=1 \mathrm{~kg}^{\mathrm{dm}}{ }^{-3} ; \mathrm{v}=1 \mathrm{~mm}^{2} \cdot \mathrm{s}^{-1} ; \mathrm{T}=20^{\circ} \mathrm{C}$; diferentes conteúdos de gás em \%) 


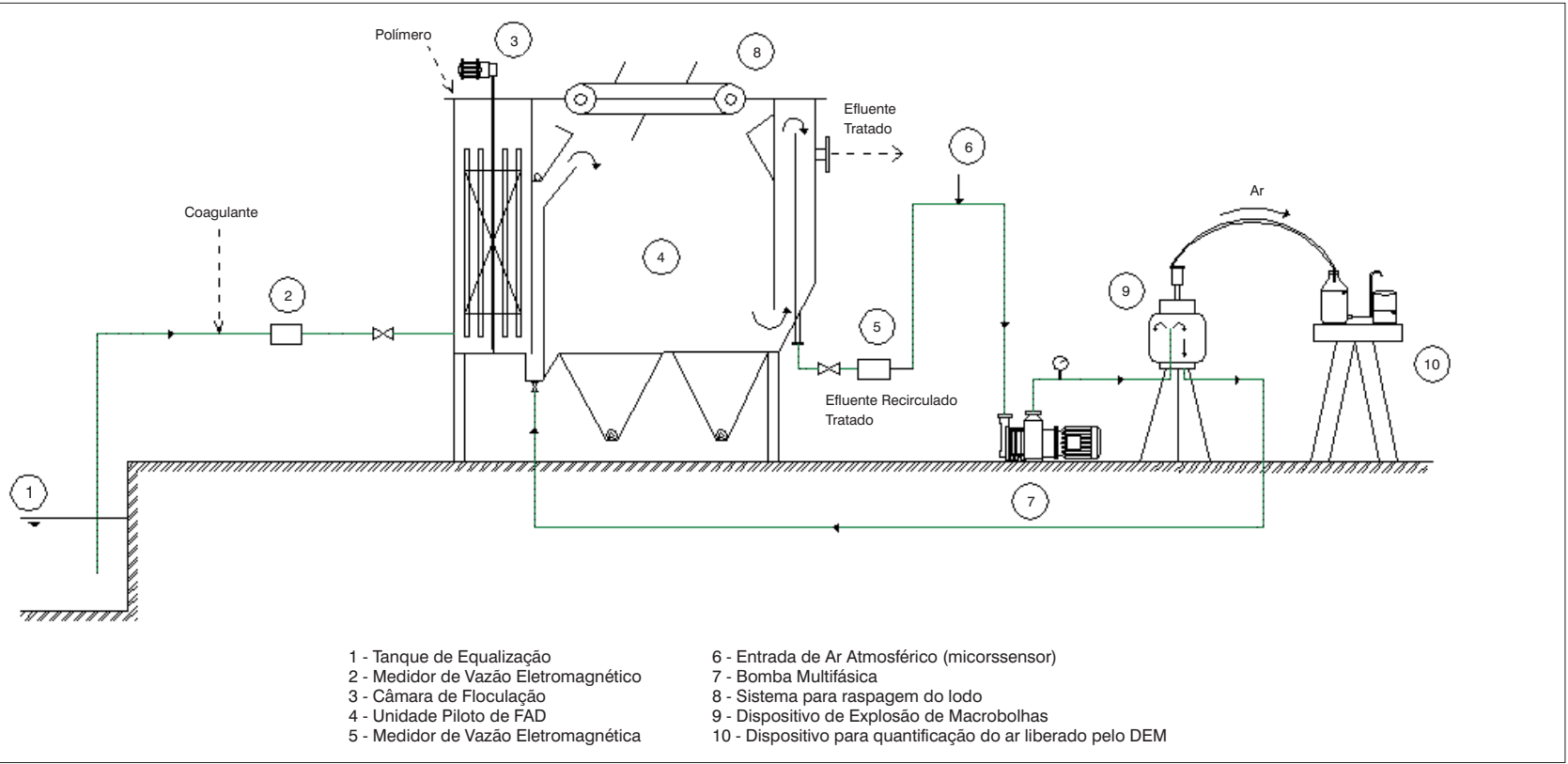

Figura 4 - Layout da instalação e seus principais componentes

\section{Determinação da quantidade de ar liberada pelo DEM durante a operação do sistema}

Durante a operação do sistema de geração de microbolhas em estudo, observou-se nos ensaios realizados a perda de ar por meio da ventosa localizada na parte superior do DEM. Assim, utilizando-se outro dispositivo especialmente desenvolvido, foi possível a quantificação do ar liberado. O dispositivo desenvolvido foi composto por um recipiente de vidro (volume igual a 2,5 L), o qual era conectado por uma mangueira à saída da ventosa e um béquer (volume igual a 2 L). No início da operação do sistema, o recipiente com vidro encontrava-se completamente preenchido com água. Desta forma, à medida que o ar fosse sendo liberado pela ventosa, este era conduzido por uma mangueira (devidamente vedada em suas extremidades e sem vazamentos) até a parte superior do recipiente de vidro, o qual encontrava-se no mesmo nível da ventosa. $\mathrm{O}$ ar liberado ao ocupar o espaço anteriormente preenchido com água deslocava esta última, a qual era coletada durante determinado intervalo de tempo por meio de um béquer. Desta forma, quantificava-se o volume de água deslocada para o interior do béquer, se determinado assim, o volume de ar liberado pelo DEM durante a operação do sistema (Figura 5).

\section{Programação dos ensaios}

\section{Etapa I - Flotateste}

Os ensaios consistiram na aplicação de diferentes dosagens de coagulante ( $\mathrm{D}_{\text {COAG }}$ igual a $600,800,1.000$ e $\left.1.100 \mathrm{mg} . \mathrm{L}^{-1}\right)$ e de polímero catiônico ( $D_{\text {PoL }}$ igual a 60, 90, 120 e 130 mg.L $\left.\mathrm{L}^{-1}\right)$ ao efluente em estudo. $\mathrm{O}$ valor de $\mathrm{pH}$ do efluente foi ajustado para um valor igual

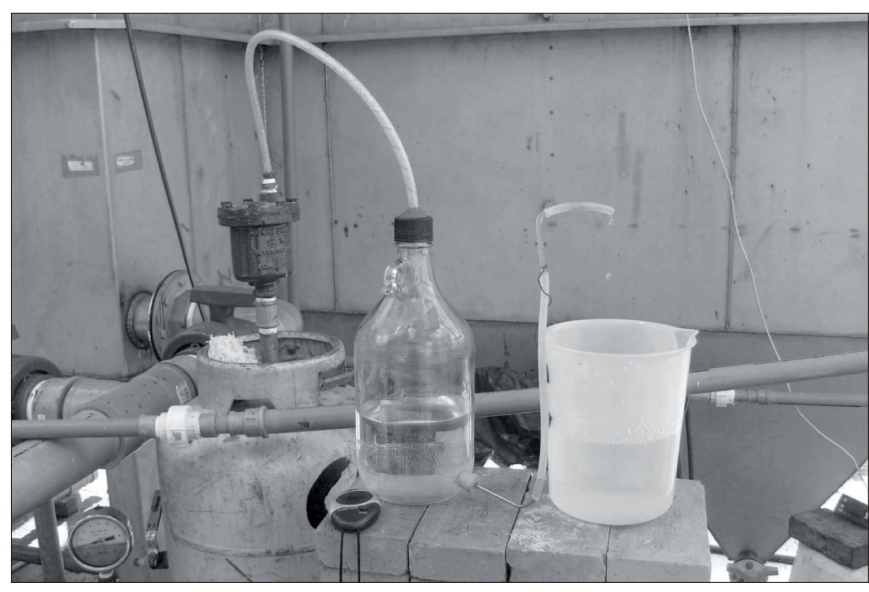

Figura 5 - Dispositivo desenvolvido para a quantificação do ar liberado durante a operação da bomba multifásica

a 9,0. Assim, simularam-se as condições e os produtos usualmente empregados no processo de tratamento de efluentes da indústria. As amostras foram coletadas em velocidade de flotação igual a 10 cm. min $^{-1}$. Em cada uma destas amostras, bem como na amostra de efluente bruto, foram realizadas medidas de turbidez e cor aparente. Para a realização destes ensaios, foi necessária a fixação dos seguintes parâmetros: (i) gradiente médio de velocidade para mistura rápida $\left(G_{M R}\right)$ de $1000 \mathrm{~s}^{-1}$; (ii) tempo médio para a mistura rápida $\left(T_{M R}\right)$ de 15 segundos; (iii) gradiente médio de velocidade para floculação $\left(G_{M L}\right)$ de $120 \mathrm{~s}^{-1}$; (iv) tempo médio de floculação $\left(\mathrm{T}_{\mathrm{ML}}\right)$ de 18 minutos; (v) pressão na câmara de saturação (p) de $550 \mathrm{kPa}$; (vi) temperatura do efluente de $25 \pm 1{ }^{\circ} \mathrm{C}$; (vii) razão de recirculação (r) de 20\%; (viii) gradiente médio de velocidade de mistura do polímero de $500 \mathrm{~s}^{-1} \mathrm{e}$ (ix) tempo médio de mistura do polímero de 15 segundos. Ressalta- 
se que o processo de mistura rápida foi efetuado em misturadores rápidos. Nestes, também ocorreu a aplicação do polímero com a finalidade de proporcionar maior mistura entre este e o efluente.

\section{Etapa II - Unidade piloto de FAD}

Os ensaios foram realizados para três diferentes razões de recirculação (20, 30 e 40\%), sendo que para cada uma destas analisou-se duas diferentes quantidades de ar fornecidas (6,5 e 13\% em volume). Os parâmetros mantidos fixos durante todos os ensaios da Etapa II foram os seguintes: i) vazão afluente $\left(\mathrm{Q}_{\mathrm{AFL}}\right)$ de $6,0 \pm 0,1 \mathrm{~m}^{3} \cdot \mathrm{h}^{-1}$; ii) gradiente médio de velocidade para floculação $\left(G_{M L}\right)$ de $280 \mathrm{~s}^{-1}$; iii) tempo médio de floculação $\left(\mathrm{T}_{\mathrm{ML}}\right)$ de 9,8 minutos; iv) dosagem de coagulante (Dos ${ }_{\mathrm{COAG}}$ ) de $1.000 \mathrm{mg} \cdot \mathrm{L}^{-1} \mathrm{e} \mathrm{v}$ ) dosagem de polímero (Dos ${ }_{\mathrm{POL}}$ ) de 120 mg. $\mathrm{L}^{-1}$. Estes dois últimos parâmetros foram determinados na Etapa I. Os seguintes parâmetros foram analisados nas amostras compostas de efluente bruto (despejo líquido industrial) e efluente clarificado (tratado): cor aparente, turbidez, demanda química de oxigênio (DQO) e sólidos suspensos totais (SST).

\section{Resultados e discussão}

\section{Etapa I - Flotateste}

Como apresentado na Tabela 1, pode-se observar que, na maior parte dos ensaios realizados no Flotateste, os valores de eficiência de remoção de turbidez e cor aparente foram superiores a 96\%, independente das dosagens de polímero e coagulante aplicadas. Devese ressaltar, entretanto, que os corantes normalmente utilizados em indústrias têxteis são resistentes à degradação biológica. Porter e Snider (1976) desenvolveram um estudo com o objetivo de analisar a biodegradação dos produtos químicos provenientes de efluentes têxteis. Os ensaios foram realizados para oito diferentes corantes, demonstrando que estes são lentamente degradados pelos micro-organismos. Os autores concluíram que o mecanismo de remoção de cor em processos biológicos ocorre por meio da adsorção dos corantes nos micro-organismos e que esta capacidade de adsorção possui um limite. Desta forma, o parâmetro que governou a escolha da dosagem adequada de coagulante e polímero foi a cor aparente, tendo em vista que o objetivo principal do pré-tratamento por flotação deste tipo de efluente era a redução da concentração de corantes antes da etapa final de tratamento biológico (lodos ativados). Assim, na unidade piloto, as seguintes dosagens foram aplicadas: $\operatorname{Dos}_{\mathrm{POL}}=120 \mathrm{mg} \cdot \mathrm{L}^{-1} \mathrm{e}$ $\operatorname{Dos}_{\mathrm{COAG}}=1000 \mathrm{mg} \cdot \mathrm{L}^{-1}$.

\section{Etapa II - Unidade piloto de FAD}

Os parâmetros de projeto utilizados nos ensaios realizados nesta etapa são apresentados na Tabela 2. Nos ensaios realizados, procurou-se manter os parâmetros taxa de aplicação superficial na zona de clarificação $\left(\right.$ TAS $_{\text {CLAR }}$ ), taxa de aplicação superficial na zona de contato $\left(\mathrm{TAS}_{\mathrm{zC}}\right)$, tempo de detenção $\left(\mathrm{T}_{\mathrm{zC}}\right)$ e velocidade na saída da zona de contato - velocidade cross-flow $\left(\mathrm{V}_{\mathrm{CF}}\right)$ fixados em faixas estreitas de valores, a saber: TAS $_{\text {CLAR }}$ em torno de 128,6 \pm 10 m.d d $^{-1}$ TAS $_{\mathrm{ZC}}$ em torno de $92,9 \pm 7 \mathrm{~m} \cdot \mathrm{h}^{-1} ; \mathrm{T}_{\mathrm{ZC}}$ em torno de 73,7 \pm 6 segundos e $\mathrm{V}_{\mathrm{CF}}$ em torno de 24,8 $\pm 2 \mathrm{~m} \cdot \mathrm{h}^{-1}$. Mantidos tais parâmetros nestas faixas, variou-se a vazão de ar $\left(Q_{A R}\right)$ e a vazão de recirculação $\left(Q_{R E C}\right)$, obtendo-se diferentes valores de pressão de operação para cada ensaio.

A Figura 6 apresenta os resultados da eficiência de remoção dos parâmetros turbidez, cor aparente, DQO e SST monitorados durante a realização dos ensaios da Etapa II.

Na Figura 6, pode-se verificar que os parâmetros cor aparente e turbidez foram os que apresentaram maiores eficiências de remoção em todos os ensaios (remoção média de 95\%), independente das condições de operação utilizadas (razão de recirculação, pressão de operação e composição volumétrica de ar). Com relação ao parâmetro DQO, este apresentou valores de eficiência de remoção na faixa de 30 a 45\%. Os maiores valores foram observados nos ensaios realizados com razão de recirculação igual a 30\% (ensaios 3 e 4), independente das composições volumétricas (Cvar) analisadas (6,5 e $13 \%$ ). Deve-se ressaltar que não constitui objetivo principal do pré-

Tabela 1 - Resultados referentes aos ensaios no Flotateste. Velocidade de flotação igual a $10 \mathrm{~cm} \cdot \mathrm{min}^{-1}$. Despejo líquido industrial: pH igual a 9,0; cor igual a $25.700 \mathrm{uH}$; turbidez igual a 2.400 NTU.

\begin{tabular}{|c|c|c|c|c|c|}
\hline \multirow{2}{*}{$\begin{array}{l}\operatorname{Dos}_{\mathrm{POL}} \\
\left(\mathrm{mg} \cdot \mathrm{L}^{-1}\right)\end{array}$} & \multirow{2}{*}{$\begin{array}{l}\text { Dos }_{\text {COAG }} \\
\left(\mathrm{mg}^{\left.-L^{-1}\right)}\right.\end{array}$} & \multirow{2}{*}{$\begin{array}{c}\text { Turbidez } \\
\text { (NTU) }\end{array}$} & \multirow{2}{*}{$\begin{array}{l}\text { Cor } \\
\text { (uH) }\end{array}$} & \multicolumn{2}{|c|}{ Eficiência (\%) } \\
\hline & & & & Turbidez & Cor \\
\hline \multirow{3}{*}{60} & 600 & 86,2 & 1860 & 96,4 & 92,8 \\
\hline & 800 & 90,6 & 552 & 96,2 & 97,8 \\
\hline & 1000 & 76,0 & 528 & 96,8 & 97,8 \\
\hline \multirow{3}{*}{90} & 600 & 56,3 & 624 & 97,6 & 97,6 \\
\hline & 800 & 56,9 & 360 & 97,6 & 98,6 \\
\hline & 1000 & 55,8 & 240 & 97,7 & 99,1 \\
\hline \multirow{3}{*}{120} & 600 & 55,4 & 324 & 97,7 & 98,7 \\
\hline & 800 & 52,4 & 276 & 97,8 & 98,8 \\
\hline & 1000 & 26,8 & 72 & 98,9 & 99,7 \\
\hline \multirow{3}{*}{130} & 1100 & 38,4 & 162 & 98,4 & 99,4 \\
\hline & 1000 & 38,0 & 156 & 98,4 & 99,4 \\
\hline & 1100 & 30,4 & 144 & 98,7 & 99,4 \\
\hline
\end{tabular}


tratamento físico-químico desse tipo de efluente (têxtil), a obtenção de elevados valores de eficiência da remoção de DQO. Na etapa de pré-tratamento, o objetivo maior é a redução máxima possível de cor do efluente, tendo em vista a etapa subsequente de tratamento biológico. Nesta, espera-se a obtenção de eficiências elevadas de remoção de DQO. A respeito da eficiência de remoção dos SST, observa-se que os maiores valores foram alcançados nos ensaios realizados com composição volumétrica de ar de 13\% (ensaios 2, 4 e 6), sendo que o ensaio $4\left(\mathrm{Cv}_{\text {ar }}\right.$ de $13 \%$ associado à pressão de $460 \pm 20 \mathrm{kPa}$ na saída da bomba multifásica e razão de recirculação de 30\%) promoveu uma eficiência de remoção de 89\%.

De maneira geral, considerando a taxa de aplicação superficial empregada neste estudo, a qual foi mantida em faixa estreita de valor (TAS $_{\text {CLAR }}$ em torno de $128,6 \pm 10 \mathrm{~m} \cdot \mathrm{d}^{-1}$ ), verificou-se que os melhores resultados de eficiência de remoção dos parâmetros monitorados foram obtidos nos ensaios realizados com razão de recirculação igual a $30 \%$ e composição volumétrica de ar $\left(\mathrm{Cv}_{\mathrm{ar}}\right)$ fornecida igual a $13 \%$.

Assim, foram verificados valores satisfatórios de eficiência de remoção dos parâmetros monitorados durante a realização da pesquisa, já que tratou-se de investigação sobre a etapa de pré-tratamento físico-químico por flotação, o qual antecede a etapa final de tratamento biológico por lodo ativado seguido de polimento final em sistema de flotação adicional.

\section{Avaliação da concentração de ar presente na recirculação pressurizada}

A concentração de ar presente na recirculação pressurizada $\left(\mathrm{C}_{\mathrm{AR}}\right)$ do sistema operado com a bomba multifásica foi avaliada em

Tabela 2 - Parâmetros de projeto empregados durante os ensaios na unidade piloto de FAD $\left(Q_{\text {AFL }}=6,0 \pm 0,1 \mathrm{~m}^{3} \cdot \mathrm{h}^{-1} ; \mathrm{D}_{\mathrm{COAG}}=1.000 \mathrm{mg} \cdot \mathrm{L}^{-1} ; \mathrm{D}_{\mathrm{POL}}=\right.$ $\left.120 \mathrm{mg} \cdot \mathrm{L}^{-1}\right)$

\begin{tabular}{|c|c|c|c|c|c|c|c|c|}
\hline Ensaio & $\begin{array}{c}r \\
(\%)\end{array}$ & $\begin{array}{c}Q_{\mathrm{REC}} \\
\left(\mathrm{m}^{3} \cdot \mathrm{h}^{-1}\right)\end{array}$ & $\begin{array}{l}\text { TASzc } \\
\left(\mathrm{m} \cdot \mathrm{h}^{-1}\right)\end{array}$ & $\begin{array}{l}\text { Tzc } \\
\text { (s) }\end{array}$ & $\begin{array}{l}\text { TAS }_{\text {CLAA }} \\
\left(\mathrm{m}^{-1} \mathrm{~d}^{-1}\right)\end{array}$ & $\begin{array}{c}V_{C F} \\
\left(m \cdot h^{-1}\right)\end{array}$ & $\begin{array}{c}Q_{\mathrm{AR}} \\
\left(\mathrm{L} \cdot \mathrm{h}^{-1}\right)\end{array}$ & $\begin{array}{c}\mathrm{p} \\
(\mathrm{kPa})\end{array}$ \\
\hline 1 & \multirow{2}{*}{40} & \multirow{2}{*}{2,4} & \multirow{2}{*}{100} & \multirow{2}{*}{68,4} & \multirow{2}{*}{138,5} & \multirow{2}{*}{26,7} & 154 & $300 \pm 20$ \\
\hline 2 & & & & & & & 307 & $100 \pm 20$ \\
\hline 3 & \multirow{2}{*}{30} & \multirow{2}{*}{1,8} & \multirow{2}{*}{92,9} & \multirow{2}{*}{73,7} & \multirow{2}{*}{128,6} & \multirow{2}{*}{24,8} & 116 & $630 \pm 20$ \\
\hline 4 & & & & & & & 230 & $460 \pm 20$ \\
\hline 5 & \multirow{2}{*}{20} & \multirow{2}{*}{1,2} & \multirow{2}{*}{85,7} & \multirow{2}{*}{79,8} & \multirow{2}{*}{118,7} & \multirow{2}{*}{22,9} & 78 & $750 \pm 20$ \\
\hline 6 & & & & & & & 154 & $660 \pm 20$ \\
\hline
\end{tabular}

* r: razão de recirculação; $Q_{\mathrm{REC}}$ : vazão de recirculação; $\mathrm{TAS}_{\mathrm{ZC}}$ : taxa de aplicação superficial na zona de contato; $T_{Z \mathrm{C}}$ : tempo de detenção na zona de contato; TAS $\mathrm{CLAR}_{\text {: }}$ taxa de aplicação superficial na zona de clarificação; $V_{\mathrm{CF}}$ : velocidade cross-flow; $\mathrm{Q}_{\mathrm{AR}}$ : vazão de ar fornecida à bomba; $\mathrm{p}$ : pressão de operação lida no manômetro instalado logo à jusante da bomba multifásica.

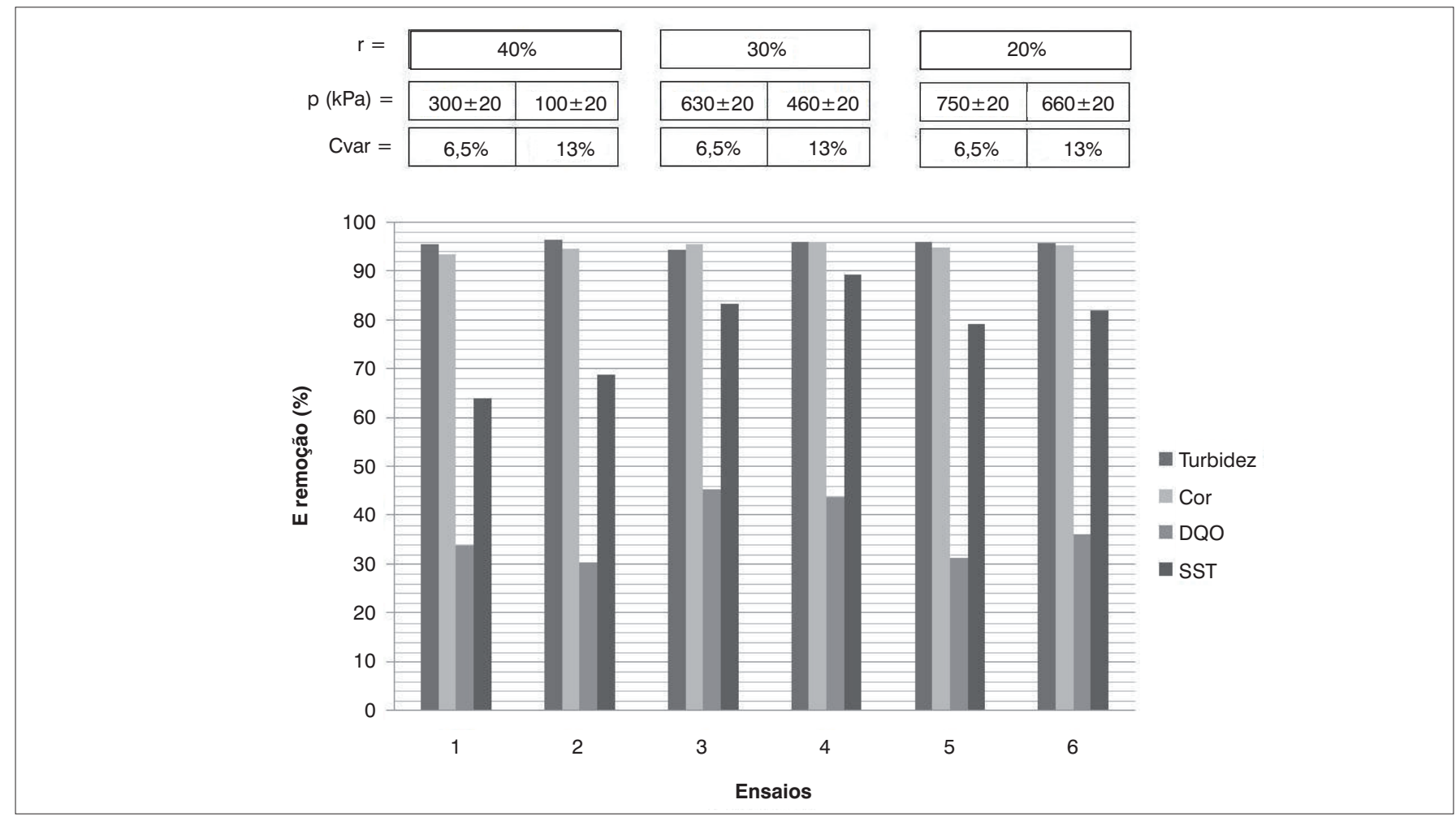

Figura 6 - Eficiências de remoção dos parâmetros monitorados durante a realização dos ensaios de 1 a $6 . Q_{A F L}$ igual $6,0 \pm 0,1 \mathrm{~m}^{3} . \mathrm{h}^{-1} ; \mathrm{TAS}_{\mathrm{CLAR}}$ na faixa

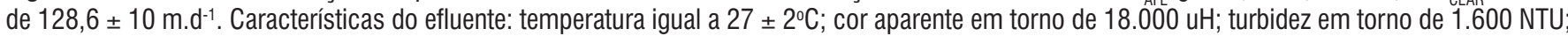
DQO em torno de $3.700 \mathrm{mgO}_{2} \mathrm{~L}^{-1}$; SST em torno de $1.200 \mathrm{mg} \cdot \mathrm{L}^{-1}$ 
comparação à concentração de ar teórica, formulada a partir da Lei de Henry para os ensaios 2, 4 e 6 (ensaios com composição volumétrica de ar fornecida igual a 13\%). Assim, a concentração de ar presente no efluente tratado e recirculado com a utilização da bomba multifásica foi calculada a partir da Equação 1; a concentração máxima teórica de ar, segundo a Lei de Henry, foi calculada a partir da Equação 2, proposta por Eckenfelder (1980); e os valores da relação entre a massa de ar efetivamente fornecida e a massa de sólidos suspensos afluente ao flotador (relação A/S) foram calculados a partir da Equação 3.

$$
C_{A R}=\frac{Q_{A R}}{Q_{R E C}} \times d_{A R} \times 1000
$$

Equação 1

Onde:

$\mathrm{C}_{\mathrm{AR}}$ : concentração total (dissolvido ou não) de ar efetivamente presente na recirculação $\left(\mathrm{mg}_{\mathrm{AR}} \cdot \mathrm{L}_{\mathrm{efl}}{ }^{-1}\right)$;

$\mathrm{Q}_{\mathrm{AR}}$ : vazão efetiva de ar fornecida ao sistema $\left(\mathrm{L} \cdot \mathrm{h}^{-1}\right)$, igual à diferença entre a vazão de ar injetada na sucção da bomba multifásica e a vazão de ar expulsa pela ventosa do DEM;

$\mathrm{Q}_{\mathrm{REC}}$ vazão de recirculação $\left(\mathrm{L} \cdot \mathrm{h}^{-1}\right)$;

$\mathrm{d}_{\mathrm{AR}}$ : densidade do ar (igual a $1,17 \mathrm{~g} \cdot \mathrm{L}^{-1}$ para $\mathrm{T}=25^{\circ} \mathrm{C}$ ).

$$
C_{A R_{\text {TEORRCA }}}=S_{A R} \times[f \times(p+1)-1]
$$

Equação 2

Onde:

$\mathrm{C}_{\mathrm{AR} \text { TEÓRICA}}$ : concentração de ar na recirculação pressurizada $\left(\mathrm{mg}_{\mathrm{AR}}\right.$. $\left.\mathrm{L}_{\text {efl }}{ }^{-1}\right)$;

$\mathrm{S}_{\mathrm{AR}}$ : solubilidade do ar na água (igual a 20,9 $\mathrm{mg}_{\mathrm{AR} .} \mathrm{L}_{\text {agua }}{ }^{-1}$ para $\mathrm{T}=30$ $\left.{ }^{\circ} \mathrm{C}\right)$;

f: fator de eficiência da câmara de saturação (igual a 1 para obtenção da concentração máxima segundo a Lei de Henry);

p: pressão relativa (atm).

$$
\frac{A}{S}=C_{A R} \times \frac{Q_{R E C}}{S S T \times Q_{A F L}}
$$

Onde:

A/S: relação ar-sólidos $\left(\mathrm{mg}_{\mathrm{AR}} \cdot \mathrm{mg}_{\text {sólidos }}{ }^{-1}\right)$;

$\mathrm{C}_{\mathrm{AR}}$ : concentração de ar na recirculação pressurizada $\left(\mathrm{mg}_{\mathrm{AR}} \cdot \mathrm{L}_{\mathrm{efl}}{ }^{-1}\right)$;

$\mathrm{Q}_{\mathrm{REC}}$ : vazão de recirculação $\left(\mathrm{m}^{3} \cdot \mathrm{h}^{-1}\right)$;

$\mathrm{Q}_{\mathrm{AFL}}$ : vazão afluente $\left(\mathrm{m}^{3} \cdot \mathrm{h}^{-1}\right)$;

SST: $\mathrm{mg} \cdot \mathrm{L}^{-1}$.

Os resultados referentes à quantidade de ar liberada pela ventosa localizada na parte superior do DEM podem ser visualizados na Tabela 3.
Pode-se verificar que em todos os ensaios a quantidade de ar liberada pelo DEM foi inferior a 1\% comparada à quantidade de ar fornecida para a realização do processo.

A concentração de ar presente no sistema constituído pela bomba multifásica (referente à vazão efetiva de ar) em comparação à concentração de ar teórica formulada a partir da Lei de Henry pode ser visualizada na Figura 7a. A relação ar-sólidos (A/S) total fornecida ao sistema de flotação em estudo (neste caso, "A" é a concentração mássica total efetivamente fornecida ao processo, envolvendo as frações de ar dissolvido e não-dissolvido), em comparação à relação arsólidos teórica (A/S) teórica, calculada a partir da Lei de Henry (neste caso, "A" é a concentração mássica máxima teórica de ar capaz de ser dissolvido na água para uma determinada pressão e temperatura), é apresentada na Figura $7 \mathrm{~b}$.

Analisando-se a Figura 7a, observa-se que, nos ensaios 2, 4 e 6, as concentrações de ar efetivamente fornecidas (fração dissolvida e não-dissolvida) pelo sistema constituído pela bomba multifásica foram superiores às concentrações máximas teóricas de ar dissolvido calculadas a partir da Lei de Henry.

No ensaio 4 (razão de recirculação de 30\% associado a um fornecimento de ar de 13\%), no qual foram verificados os melhores resultados em termos de eficiência de remoção dos parâmetros analisados, a concentração máxima teórica de ar dissolvido (Lei de Henry) foi 37\% inferior à concentração de ar total (fração dissolvida e nãodissolvida) fornecida para o processo e apresentou uma relação A/S em torno de 0,015 $\mathrm{g}_{\mathrm{ar}} \cdot \mathrm{g}_{\text {sólidos }}{ }^{-1}$ (Figura 7b).

Assim, pode-se concluir que as bolhas de ar fornecidas pela bomba multifásica foram partes oriundas do ar previamente dissolvido e partes oriundas da ruptura de bolhas maiores no interior da bomba multifásica (parcela de ar não-previamente dissolvido). De qualquer forma, independente de como foi gerado, esse conjunto de bolhas de ar presente na recirculação pressurizada (após o DEM) foi capaz de promover, satisfatoriamente, a separação dos sólidos em suspensão na unidade de flotação.

Finalmente, vale ressaltar que a bomba multifásica utilizada nas condições deste estudo não produziu quantidade exagerada de bolhas grandes passíveis de serem retidas e expulsas pelo DEM. Entretanto, recomenda-se a realização de estudos complementares com vistas à determinação da distribuição de tamanho de bolhas geradas pela bomba multifásica e sua influência na eficiência do pro-

\begin{tabular}{|c|c|c|c|c|c|c|}
\hline Ensaio & $\begin{array}{c}r \\
(\%)\end{array}$ & $\underset{\left(\mathrm{m}^{3} \cdot \mathrm{h}^{-1}\right)}{\mathrm{R}_{\mathrm{BEC}}}$ & $\begin{array}{c}p^{*} \\
(\mathrm{kPa})\end{array}$ & $\begin{array}{l}Q_{\text {AR FORNECIDA }} \\
\left(L^{-1} h^{-1}\right)\end{array}$ & $\begin{array}{c}Q_{\text {AR LIBERADA NA VENTOSA }} \\
\left(L . h^{-1}\right)\end{array}$ & $\begin{array}{l}Q_{\text {AR EFETIVA }} \\
\quad\left(L . h^{-1}\right)\end{array}$ \\
\hline 2 & 40 & 2,4 & $100 \pm 20$ & 307 & 1,8 & 305,2 \\
\hline 4 & 30 & 1,8 & $460 \pm 20$ & 230 & 0,9 & 229,1 \\
\hline 6 & 20 & 1,2 & $660 \pm 20$ & 154 & 1,2 & 152,8 \\
\hline
\end{tabular}
cesso de flotação.

Tabela 3 - Resultados da avaliação da quantidade efetiva de ar utilizado no processo FAD com utilização de bomba multifásica

* pressão de operação lida no manômetro instalado logo à jusante da bomba multifásica. $Q_{\mathrm{REC}}$ : vazão de recirculação; $Q_{A R}$ : vazão de ar fornecida à bomba. 


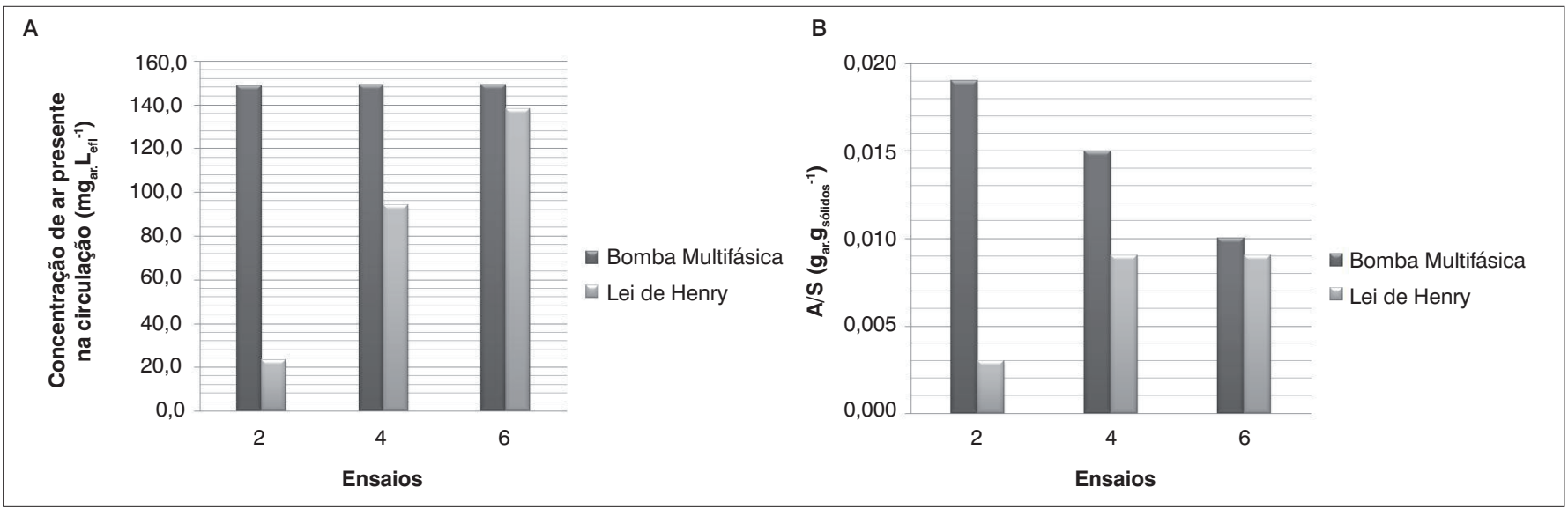

Figura 7 - (a) Quantidade de ar efetivamente fornecida ao sistema em estudo em comparação à máxima quantidade teórica de ar dissolvido calculada de acordo com a Lei de Henry; (b) relação A/S fornecida ao sistema em estudo em comparação à relação $A / S$ teórica

\section{Conclusões}

Considerando a taxa de aplicação superficial empregada neste estudo, a qual foi mantida em faixa estreita de valor (TAS ${ }_{\text {CLAR }}$ em torno de $128,6 \pm 10 \mathrm{~m} \cdot \mathrm{d}^{-1}$ ), verificou-se que os maiores valores de eficiência de remoção dos parâmetros monitorados foram obtidos nos ensaios realizados com razão de recirculação igual a 30\% associado ao fornecimento de ar de 13\%.

Nos ensaios onde se realizou a avaliação da concentração de ar presente na recirculação pressurizada, as concentrações de ar efetivamente fornecidas (fração dissolvida e não- dissolvida) pelo sistema constituído pela bomba multifásica foram superiores às concentrações máximas teóricas de ar dissolvido calculadas a partir da Lei de Henry.
$\mathrm{O}$ valor da relação A/S, que forneceu os melhores resultados, situou-se em torno de 0,015 $\mathrm{g}_{\mathrm{ar}} \cdot \mathrm{g}_{\text {sólidos }}{ }^{-1}$. Desta forma, sugere-se tal valor como sendo o mínimo necessário para a operação do sistema de flotação investigado.

Sugere-se a comparação entre o sistema de geração de microbolhas por bomba multifásica com o sistema tradicional com câmara de saturação. Tal comparação poderá ser realizada pela investigação experimental com a utilização de instalação piloto, como aquela empregada no presente estudo, alimentada com o mesmo tipo de efluente. Por meio desta investigação comparativa, será possível a obtenção de dados concernentes ao consumo de energia e eficiência de clarificação por flotação para cada um dos sistemas citados.

\section{Referências}

ECKENFELDER, W. W. Principles of Water Quality Management. Boston, USA: CBI Publishing Company, 1980, 360p.

HAN, M.; KIM, T.; PARK, S.; JUNG, Y. The development of a generator to produce bubbles of tailored sizes. In: The $5^{\text {th }}$ International Conference on Flotation in Water and Wastewater Systems, Seul, Korea, 2007.

KIURU, H. J. Development of dissolved air flotation technology from the first generation to the newest (third) one (DAF in turbulent flow conditions). Water Science and Technology, Great Britain, v. 43, n. 8, p. 1-7, 2001.

LEE, C. H.; AN, D. M.; KIM, S.S.; AHN, K. H.; CHO, S. H. Full scale operation of dissolved air flotation process using microbuble generating pump. In: The $5^{\text {th }}$ International Conference on Flotation in Water and Wastewater Systems, Seul, Korea, 2007.

PORTER, J. J; SNIDER, E. H. Long-term biodegradability of textile chemicals. Journal - Water Pollution and Control Federation, v. 48, n. 9, p. 2198-2210, 1976 .
REALI, M. A. P. Concepção e avaliação de um sistema compacto para tratamento de águas de abastecimento utilizando o processo de flotação por ar dissolvido e filtração com taxa declinante. Tese (Doutorado em Hidráulica e Saneamento) - Escola de Engenharia de São Carlos, Universidade de São Paulo, São Carlos, 1991.

ROSS, C.C.; SMITH, B.M.; VALENTINE, G.E. Rethinking dissolved air flotation (DAF) design for industrial pretreatment. In: Water Environment Federation and Purdue University Industrial Wastes Technical Conference, St. Louis, Missouri, 2000.

RUBIO, J.; SOUZA, M.L.; SMITH, R.W. Overview of flotation as a wastewater treatment technique. Minerals Engineering, Great Britain, $\mathrm{n}$. 15, p. 139-155, 2002

SOHN, B. Y. et al. Application of ozone combined with DAF process for water treatment process. In: The $5^{\text {th }}$ International Conference on Flotation in Water and Wastewater Systems, Seul, Korea, 2007. 